Théologiques

Théologiques

\title{
La conception du travail dans la Bible et dans la tradition chrétienne occidentale
}

\section{Aldina Da Silva}

Volume 3, numéro 2, octobre 1995

Crise du travail, crise de civilisation

URI : https://id.erudit.org/iderudit/602426ar

DOI : https://doi.org/10.7202/602426ar

Aller au sommaire du numéro

Éditeur(s)

Faculté de théologie de l'Université de Montréal

ISSN

1188-7109 (imprimé)

1492-1413 (numérique)

Découvrir la revue

Citer cet article

Da Silva, A. (1995). La conception du travail dans la Bible et dans la tradition chrétienne occidentale. Théologiques, 3(2), 89-104.

https://doi.org/10.7202/602426ar
Résumé de l'article

Dans la tradition chrétienne occidentale le travail fait parfois l'objet d'une appréciation positive, mais bien plus souvent négative. D'où vient cette vision péjorative attachée à l'image du travail? Pour répondre à cette question, cet article aborde, en premier lieu, la conception du travail dans la Bible à partir d'une étude du vocabulaire et d'une analyse de quelques textes de la Genèse, des Sages, des Prophètes et du Nouveau Testament. En deuxième lieu, nous montrerons comment quelques textes bibliques, souvent isolés de leur contexte, ont influencé les conceptions et pratiques historiques du christianisme à propos du travail. 
Theologiques 3/2 (1995) 89-104.

\title{
La conception du travail dans la Bible et dans la tradition chrétienne occidentale
}

\author{
Aldina DA SILVA \\ Faculté de théologie \\ Université de Montréal
}

\section{RÉSUMÉ}

Dans la tradition chrétienne occidentale le travail fait parfois l'objet d'une appréciation positive, mais bien plus souvent négative. D'où vient cette vision péjorative attachée à l'image du travail? Pour répondre à cette question, cet article aborde, en premier lieu, la conception du travail dans la Bible à partir d'une étude du vocabulaire et d'une analyse de quelques textes de la Genèse, des Sages, des Prophètes et du Nouveau Testament. En deuxième lieu, nous montrerons comment quelques textes bibliques, souvent isolés de leur contexte, ont influencé les conceptions et pratiques historiques du christianisme à propos du travail.

Le verbe «travailler» en latin signifie jusqu'au XVI ${ }^{\mathrm{e}}$ siècle «faire souffrir », « tourmenter ${ }^{1}$ ». Il vient du vocable tripalium qui désignait, dans le latin tardif, un appareil à trois pieux permettant d'immobiliser le cheval que l'on voulait ferrer. De là, on est passé au sens plus général de torture. Encore au XVIII ${ }^{e}$ siècle, le Dictionnaire de l'Académie française souligne que le travail implique un effort douloureux, souffrant et pénible $^{2}$. Les gens de travail sont des hommes de peine. Cette même idée

1 E. HUGUET, Dictionnaire de la langue française du seizième siècle. Vol. 7, Paris, Didier, 1967, p. 319-320.

2 En 1935, la huitième édition révisée de ce dictionnaire, Paris, Librairie Hachette, définit ainsi le mot travail : «labeur, effort soutenu pour faire quelque 
est contenue dans le vocable "labeur" qui provient du mot latin labor, " peine ». Il a donné ensuite laborius, " pénible". Il est aussi intéressant de remarquer que les mots "peine » et «punir» viennent de la même racine latine.

Cette simple histoire des mots illustre la perception pessimiste du travail qui traverse la culture de l'Occident. Pourtant, à côté de cette vision aliénante du travail, une autre vision parcourt la pensée chrétienne : celle du travail comme lieu de libération. Dans la première partie de cette étude nous essaierons donc de montrer comment cette ambivalence à l'égard du travail se retrouve déjà dans les textes bibliques. Pour ce faire, nous étudierons dans un premier temps le vocabulaire biblique concernant le travail. Puis, nous aborderons les conceptions optimiste et pessimiste du travail à partir de l'analyse de quelques textes de la Genèse, des Prophètes, des Sages et du Nouveau Testament. Dans la deuxième partie, nous montrerons comment quelques textes bibliques, souvent isolés de leur contexte, ont influencé les conceptions et pratiques historiques du christianisme à propos du travail. Bien entendu, nos propos concerneront surtout la tradition catholique. En plus, nous ne retiendrons que les aspects principaux de la question et nous ne pourrons pas toujours établir les nuances nécessaires, mais il ne saurait en être autrement lorsqu'on traite un sujet d'une telle ampleur dans un espace aussi restreint.

\section{Le travail dans la Bible}

\subsection{Le vocabulaire}

Le vocabulaire hébraïque connaît au moins une dizaine de substantifs pour décrire le travail ${ }^{3}$. Les mots qui désignent le travail sans connotation

chose », et le mot travailler : « tourmenter, soumettre à une gêne, causer de la peine ». Aujourd'hui encore on dit en travail une femme en douleur d'enfantement.

3 Sur le travail dans la Bible, voir : Trabalho et trabalhador, Estudos Bíblicos 11, Petrópolis, Vozes, 1986, 122 p.; C. WESTERMANN, « Travail et œuvre culturelle dans la Bible », Concilium 151 (1980), p. 95-106; N. B. PEREIRA, « O trabalho e o trabalhador na Bíblia », RCB 21 (1978) p. 79-100; P. de SURGY et J. GUILLET, art. « Travail », Vocabulaire de théologie biblique. Paris, Cerf, 1977, col. 1306-1312; G. AGRELL, Work, Toil and Sustenance. Verbum, Hakan Ohlssons, 1976, 261 p.; P. BEAUCHAMP, « Travail et non-travail dans la Bible ", LumVie 124 (1975) p. 59-70; H. W. WOLfF, Anthropologie de l'Ancien Testament. Genève, Labor et Fides, 1974, p. 112-116; L. RAMLOT, art. "Trabajo», Enciclopedia de la Biblia. Vol.6, Barcelona, Ediciones Garriga, 1965, col. 1050-1075; P. BENOIT, «Le travail selon la Bible », LumVie 20 
péjorative sont les suivants: $m$ 'sh, "action ", " ouvrage ", " travail (des champs, artistique)", "fruit du travail ", "travail (œeuvre de Dieu) "; 'bdh, "travail quotidien" qui peut être celui des champs, de la construction, ou des occupations sacerdotales incluant la liturgie; ml'kh, " mission", "travail ", " métier ", " commerce "; p'l, " travail ", " œuvre", "salaire". Les mots qui désignent le travail à connotation péjorative sont les suivants: ' $m l$, "travail » dans tout ce qu'il a de plus fatigant et pénible; $y g y^{\prime}$ englobe le sens de pénalité attachée au «travail ", la fatigue des mains; 'ṣb, "douleur », " peine (du travail) »; $m s$, « travail obligatoire », " corvée » et sblwt, « corvée ».

Les travailleurs sont souvent désignés dans la Bible par leur spécialité. Nous ne citerons ici que quelques termes généraux. Le plus fréquent est 'bd, " serviteur", "esclave ", mais aussi " sujet du roi ", «ministre » ou « haut fonctionnaire »; $h r \zeta ̌$ qui désigne toute sorte d'artisans, en particulier les métalurgistes, les fabriquants d'idoles et les charpentiers; ' $m l$, "celui qui se fatigue", ne revient que deux fois avec le sens de travailleur Ug 5,26; Qo 9,9). Pour désigner les "travailleurs", l'hébreu emploie plutôt des expressions comme 'sh ml'kh, "exécuteurs du travail " ou ' $n s$ ml'kh, "hommes du travail".

En ce qui concerne le vocabulaire grec de la Septante et du Nouveau Testament ${ }^{4}$, nous avons, sans connotation péjorative, les substantifs suivants : ergon, "action ", " œuvre " dans son sens général; poiema, " œuvre " metrant en relief l'aspect créateur de l'action; pragma, praxis, "activité ". Avec une connotation péjorative, signalons pónos, "travail" associé à la fatigue, souffrance, pauvreté et même maladie; kámatos qui exprime, plus encore que ponos, l'effort lié au travail et kopos, "peine", "souffrance ", " travail pénible ». Ce dernier terme traduit souvent dans

(1955) p. 209-222; H. LESÊTRE, art. "Travail », Dictionnaire de la Bible. Vol. 5, Paris, Letouzey et Ané, 1912, col. 2302-2305. Sur le vocabulaire hébraïque du travail on consultera aux articles respectifs les dictionnaires suivants : Theological Dictionary of the Old Testament; Theologisches Handwörterbuch zum Alten Testament. Cf. encore L. RAMLOT, art. cit., col. 1050-1052 et P. HUMBERT, « L'emploi du verbe pa'al et de ses dérivés substantifs en hébreu biblique », ZeitAltW 65 (1953) p. 35-44.

4 Sur le vocabulaire grec du travail on consultera les articles respectifs dans le dictionnaire suivant: Theological Dictionary of the New Testament. Cf. aussi F. GRYGLEWICZ, « La valeur morale du travail manuel dans la terminologie grecque de la Bible », Biblica 37 (1956) p. 314-337; L. RAMLOT, art. cit., col. 1070-1071; H..C. HAHN et al., art. "Trabalhar, Fazer, Cumprir ", Dicionário International de Teologia do Novo Testamento. Vol. IV, Sao Paulo, 1983, p. 643-655. 
la Septante le mot ' $m l$ et forme parfois avec ponos un hendiadys (voir par exemple Jr 20,18; Ps 90,10).

Cette analyse rapide des substantifs servant à décrire le travail dans la Bible (on pourrait en faire autant pour les verbes et adjectifs) nous montre déjà comment cette réalité est ambiguë. Cependant une première nuance s'impose: le vocabulaire qui précise le caractère pénible et souffrant du travail ne revient qu'exceptionnellement.

\subsection{Conception optimiste $d u$ travail}

\section{Les textes de la Genèse}

Dans la Bible, «le travail constitue une part essentielle du discours qui parle du Créateur et de la création ${ }^{5}$ ". À la fin de son récit imagé de la création en six jours, l'auteur biblique conclut: "Dieu acheva au septième jour l'œuvre ( $m l ' k h)$ qu'il avait faite, il arrêta au septième jour toute l'œuvre ( $m l ' k h)$ qu'il faisait » (Gn 2,2; cf. Ps 8,2;102,26)6. C'est cette activité créatrice que chaque personne, comme "image" de Dieu, est appelée à continuer :

Dieu dit: "Faisons l'être humain à notre image, selon notre ressemblance, et qu'il domine sur les poissons de la mer, sur les oiseaux du ciel, sur les bestiaux, sur toutes les bêtes sauvages et sur tous les reptiles qui rampent sur la terre. » $(G n 1,26)$

Cet ordre de Dieu donné à l'être humain de dominer les animaux et la terre suppose la loi du travail, même si elle n'y est pas formellement exprimée.

Quant au deuxième récit de la création, il nous présente l'être humain comme "co-créateur». En effet, la terre nous est ici décrite comme un immense désert aride sans aucun signe de vie. L'expression est claire : "il n'y avait pas d'être humain (' $d m$ ) pour travailler ('bd) la terre ('dmh)" (Gn 2,5b). Ce texte établit ainsi un lien entre la création de l'être humain et la culture du sol: sa fonction première est de transformer en terre

5 J.-J. LAVOIE, La pensée du Qohélet. Etude exégétique et intertextuelle. Montréal, Fides, 1992, p. 197.

6 Partout, dans la Bible, Dieu lui-même est au travail. Il est présenté soit comme un Architecte $\left(P_{s} 104,2-5\right)$, un Cultivateur $\left(P_{s} 65,10-14 ; 104,13-14\right)$, un Vendangeur ( $I s$ 63,3), ou un Potier (Gn 2,7; Is 45,9-12; 64,7; Ir 18,6). 
arable le désert stérile ${ }^{7}$. La suite du récit apporte des précisions. Le travail humain se déroule à l'intérieur d'un jardin que Dieu a lui-même planté $\left(n t^{\prime}\right)(G n$ 2,8-9). Dieu confie alors à l'être humain la mission de cultiver ('bd) et de garder $(\xi m r)$ ce jardin $(G n 2,15)$, deux verbes qui expriment des actions complémentaires caractéristiques du travail agricole. En lui confiant les richesses de sa création, Dieu charge l'être humain d'une grande responsabilité : celle de l'élaboration et de la conservation de ces biens. Ainsi, en Israël, l'être humain est creé "travailleur". Le travail fait partie de sa condition humaine et ne présente aucune connotation négative. Pourtant, ce récit lance un premier avertissement : tout travail qui ne vise que le bénéfice sans considérer la garde et le soin du sol est considéré comme étant contraire à l'ordre du Créateur.

Au chapitre 4 de la Genèse, tout de suite après l'expulsion de l'être humain du jardin, le texte nous met en présence de différentes professions : agriculteur et éleveur de petit bétail $(4,2)$, musiciens $(4,21)$ et forgerons $(4,22)$. Nous avons ici une description optimiste des multiples facettes du travail humain qui conduit au développement. Celui-ci ne peut être que le résultat de la bénédiction. Pourtant, le climat de violence qui accompagne l'apparition de la division du travail et de l'augmentation technique, à savoir l'homicide de Caïn et la vengeance de Lamech, laisse sous-entendre un deuxième avertissement : le progrès de la technique peut favoriser une certaine puissance qui menace la communauté humaine (cf. aussi $\mathrm{G} n$ 11,1-9).

\section{Les textes sapientiaux et le psautier}

Le problème du travail et de son succès a fait l'objet de plusieurs réfléxions favorables de la part des sages d'Israël. Tout d'abord ils établissent l'équation suivante : le travail engendre le succès. L'homme travailleur obtient la compensation de son effort. De la même façon, la liberté, une bonne position sociale ou le bonheur, dépendent de la manière dont on s'astreint au travail : "la main diligente enrichit " $(\mathrm{Pr}$ $10,4 \mathrm{~b})$; "les mains actives commanderont " $(\operatorname{Pr} 12,24 \mathrm{a})$; « les calculs de l'homme actif sont un profit assuré " (Pr 21,5a); quelqu'un d'habile dans ce

Nous trouvons cette même fonction première de l'être humain dans les textes mésopotamiens. Cependant, en Israël, cette fonction ne répond pas à un besoin divin comme c'est le cas en Mésopotamie. Sur cette question cf. G. COUTURIER, « La mort en Mésopotamie et en Israël : phénomène naturel ou salaire du péché? », dans Essais sur la mort. Montréal, Fides, 1985, p. 51-98. 
qu'il fait peut "se présenter devant les rois au lieu de rester parmi les gens obscurs " $(\operatorname{Pr} 22,29)$; " tu te nourris du labeur de tes mains, heureux es-tu! À toi le bonheur! "(Ps 128,2). D'où la condamnation fréquente de l'oisiveté, car si l'assiduité au travail procure l'abondance et la dignité, l'indolence engendre la pauvreté et la servitude : "la main indolente appauvrit " $(\operatorname{Pr} 10,4 a)$ et "la paresse mène au travail forcé " $(\operatorname{Pr} 12,24 b)$. Pourtant, l'expérience de la vie indique que le succès du travail est discutable. La diligence humaine ne mène pas forcément à la réussite ( 0 11,6). Il y a des travailleurs assidus qui restent pauvres et manquent de tout. Cette inutilité de l'effort humain tient à des circonstances malheureuses, voire à l'absence de la bénédiction divine. Le sage proclame : "c'est la bénédiction du Seigneur qui enrichit et le tourment n'y ajoutera rien " $(\operatorname{Pr} 10,22)$. Or, la bénédiction est le résultat de la fidélité à Dieu. Celui qui n'est pas fidèle voit le fruit de son travail passer aux mains des autres $(\operatorname{Pr} 5,10 \text {; cf. Ps } 109,11 ; D t 28,33)^{8}$. Le psalmiste ajoute :

Si le Seigneur ne bâtit pas la maison, ses bâtisseurs travaillent pour rien. Si le Seigneur ne garde la ville, la garde veille pour rien. Rien ne sert de vous lever tôt, de retarder votre repos, de manger un pain pétri de peines! $\grave{A}$ son ami qui dort, il donnera tout autant (Ps 127,1-2)

Ce psaume ne fait aucunement la promotion de la paresse. Il entend plutôt rappeler que le succès lié au travail dépend de Dieu seul (cf. aussi Dt 8,17-18).

\subsection{Conception pessimiste du travail}

\section{Les textes de la Genèse}

Les quelques exemples que nous venons de considérer permettent de conclure que dans la Bible, d'une manière générale, le travail est vu comme une activité naturelle et joyeuse. Pourtant, l'expérience de la vie quotidienne montre que, malgré la joie qui est ratrachée au travail, celui-ci est souvent bien ardu. Ce n'est pas sans un pénible effort que les agriculteurs arrachent au sol aride de la Palestine les quelques fruits pour se nourrir. Le texte de $G n$ 3,17-19, qui a servi pendant longtemps d'explication au caractère souffrant du travail, fait écho à cette réalité :

Cetre même idée est développée par le Deutéronome. Le malheur de celui qui brise l'Alliance consiste à voir le produir de son travail mangé par un peuple étranger (Dt 28,30-33; cf. Am 9,14; Is 62,8-9). Par contre, la fécondité du sol dépend de la fidélité à l'Alliance (Dt 28,12; cf. 14,29; 16,15). 
(Dieu) dir à Adam : [...] le sol sera maudit ('rr) à cause de toi. C'est dans la peine ('sb) que tu t'en nourriras tous les jours de ta vie, il fera germer pour toi l'épine et le chardon et tu mangeras l'herbe des champs. À la sueur de ton visage tu mangeras du pain jusqu'à ce que tu retournes au sol car c'est de lui que tu as été pris. Oui, tu es poussière et à la poussière tu retourneras.

Il ne s'agit pas de voir dans ces versets une malédiction sur le travail. En effet, ni Adam, ni le travail de ses mains sont maudits. Seule la terre est maudite. La nature de cette malédiction est décrite immédiatement après : «il fera germer pour toi ronce et épine». Dorénavant, la production de la nourriture sera le résultat d'un travail pénible «à la sueur de ton front ». Pourtant, nous ne sommes pas ici en présence d'un destin fatal et irrémédiable. Au Déluge succède une alliance nouvelle avec Noé et sa descendance. Dieu rend alors la terre à l'homme comme source permanente de fécondité (Gn 8,21-22).

\section{Les textes prophétiques}

$\mathrm{Ce}$ qui intéresse les prophètes, c'est surtout la justice sociale. Toute œuvre née au prix de l'oppression et de l'injustice ne peut pas être acceptée (cf. Hab 2,11-12).

Les accusations contre les riches qui se livtent à tous les plaisirs et vivent dans le luxe acquis par l'exploitation des petites gens sont nombreuses (Am 6,3-6; Is 1,17; 5,8; etc). Elles visent non seulement les conditions de travail, mais aussi le salaire. $\grave{A}$ ce sujet les propos de Jérémie sont clairs :

Malheureux celui qui construit son palais au mépris de la justice, et ses étages au mépris du droit; qui fait travailler (' $b d$ ) les autres pour rien, sans leur donner de salaire ( $\left.p^{\prime} l w\right)$ or 22,13).

La loi deutéronomique va même plus loin en déterminant que le travailleur doit être payé le jour même de son service (Dt 24,14-15; cf. aussi $L v 19,13$ ).

Cependant on aura remarqué que les critiques des prophètes ne portent pas sur le travail comme tel. Ils s'insurgent plutôt contre l'arbitraire, l'injustice, la violence et la rapacité qui font du travail un poids accablant. 


\section{Les textes sapientiaux}

Pour le Qohélet la valeur du travail est bien relative ${ }^{9}$. Certes, il a pu jouir de son labeur $(2,10 \mathrm{~d})$, mais finalement "les œuvres qui se font sous le soleil » ne sont qu'absurdité $(1,14 ; 2,11)$.

Si le Qohélet a une conception si négative du travail, c'est que celui-ci, en plus d'être aliéné et aliénant $(4,4-6)$, rappelle constamment la finitude humaine $(11,6)$. En bref, le Qohélet ne nie pas totalement la valeur du travail, mais il ne saurait être question de chercher un sens à sa vie dans celui-ci.

\subsection{Le Nouveau Testament}

Les évangiles signalent que Jésus vient d'un atelier de charpenterie (Mc 6,3; cf. Mt 13,55) et ses principaux disciples d'une entreprise de pêche $(M t 4,18)$. Ces travaux ne sont pas valorisés comme tels et le silence des évangiles par rapport au travail est étonnant. 11 reste cependant que le Nouveau Testament se situe spontanément dans un monde où le travail est le lot quotidien des humains. Nous n'avons qu'à penser au langage métaphorique que les évangiles empruntent au monde du travail pour décrire la tâche de Jésus et celle de ses disciples. On parle du berger (Jn $10,1)$, du vigneron $($ In 15,1), du semeur (Mc 4,3), du moissonneur (Mt 9,37), du pêcheur (Mc 1,17), du marchand (Mt 13,45), de la ménagère ( $L c 15,8$ ), de la femme qui pétrit le pain $(M t 13,33)$, des serviteurs et des servantes qui font de la table un lieu festif (Mt 22,3-4).

Par ailleurs, les évangiles condamnent la surestimation du travail. Dans la parabole du riche cultivateur Jésus rejette l'opinion très largement répandue que le travail peut assurer la vie : "Insensé, cette nuit même, on va te redemander ta vie » $(L c 12,20)$. Le Sermon sur la montagne rejette lui aussi le souci de la vie :

Regardez les oiseaux du ciel : ils ne sèment, ni ne moissonnent, ni ne ramassent dans des greniers, et votre Père céleste les nourrit! $\mathrm{Ne}$ valez-vous pas, vous, beaucoup plus qu'eux? [...] Et du vêtement pourquoi être en souci? Observez les lis des champs, comme ils croissent: ils ne peinent ni ne filent. Or je vous dis que pas même Salomon, dans toute sa gloire, n'a été vêtu comme l'un d'eux (Mt 6,2629; cf. Lc 12,22-24).

9 Sur cette question cf. J.J. LAVOIE, « À quoi sert-il de perdre sa vie à la gagner? Le repos dans le Qohelet", ScEsp XIV 13 (1992) p. 331-347 et N. B. PEREIRA, «O sentido do trabalho nos livros sapienciais », EstBib 11 (1986) p. 83-86. 
Il ne s'agit pas ici de dévaloriser le travail, mais plutôt de le situer à sa juste place. À ce sujet, l'exemple de Paul est éloquent. Allant à l'encontre d'une conception grecque classique qui réservait le travail manuel à l'esclave, Paul affirme que le travail physique est une obligation à laquelle aucun individu ne saurait s'exempter. En cela il se situe dans la ligne de la pensée juive qui associe au travail intellectuel l'exercice d'un métier. Lui-même travaille de ses mains (Ac 18,13; 20,34; 1 Co 4,12), jour et nuit, afin de n'être à la charge de personne ( 1 Th 2,9; 2 Th 3,8). À ceux qui sont tentés par l'oisiveté, Paul leur recommande "dans le Seigneur Jésus Christ de travailler tranquilles et de manger le pain qu'ils auront eux-mêmes gagné » (2 Th 3,12).

Cette étude sur le travail dans la Bible a mis en relief la prééminence du caractère " optimiste » sur le caractère " pessimiste » dans l'appréciation du travail. Il nous faut maintenant examiner comment ces textes bibliques ont été repris, commentés et interprétés par la tradition chrétienne.

\section{Le travail dans la tradition chrétienne et l'Occident ${ }^{10}$}

\subsection{L'époque médiévale}

Sur la question du travail, les textes bibliques les plus commentés au Moyen Âge sont ceux qui envisagent son caractère pénible, tout particulièrement Gn 3,17-18. L'interprération qu'on donne de ce passage fait alors du travail une

10 Voir à ce sujet « Le travail entre sens et non-sens », LumVie 220 (1994) p. 1-63; P. VALLN, «Les spiritualités du travail. Un nouveau chapitre? », Christus 161 (1994) p. 57. 67; J. RICHARD et L. ONEIII (dir.), La question saciale hier et argorad' hui. Colloque du centenaire de Rerum Novarum. Sainte-Foy, Presses de l'Université Laval, 1993; R. ANTONCICH et J. M. Munarriz, La doctrine sociale de l'Église. Paris, Cerf, 1992, p. 110-227; G. BauM, « L'originalité de l'enseignement social catholique ", Concilium 237 (1991) p. 65-74; J. M. DiEz-ALEGRIA, « Propriété et travail. Développement de l'enseignement pontifical „, Concilizan 237 (1991) p. 33-39; J. Hamesse er C. MuRAIlle-Samaran (dir.), Le travail au Moyen Âge. Une approche interdisciplinaire. Louvain-La-Neuve, 1990; J. G. TREMBLAY, Le travail en quête de sens. Montréal, Paulines, 1990; M. SiLvA, « Le monde du travail en changement », dans Travail, Culture, Religions. Fribourg, Éditions universitaires, 1988, p. 101-119; E. BARBOTIN, «Travail, péché, salut *, Commumio $9 \backslash 2$ (1984) p. 16-15; J. VALARCHE, * La doctrine pontificale du travail », Communio $9 \backslash 2$ (1984) p. 48-58; P. VALLN, Le travail et les travailleurs dans le monde chrétien. Paris, Desclée, 1983; I. Ellacuria, « Le Royaume de Dieu et le chômage dans le Tiers Monde », Concilium 180 (1982) p. 149-158; G. PIANA, « Travail humain, bénédiction et \ou malédiction? ", Concilium 180 (1982) p. 109-120; R. LATOURE LE, L'homme et ses problèmes dans la lumière du Christ. Montréal, Bellarmin, 1981, p. 255-278; S. BURGalassi, * Pour une théologie de I'homme en tant que travailleur ", Concilirm 151 (1980) p. 120-133; F. SCHUSSlER FIORENZA, « Croyances religieuses et praxis. Réflexions sur 
malédiction et un châtiment du péché. Déjà l'augustinisme affirmait que le travail n'avait de valeur que par « l'acceptation pénitentielle de sa souffrance ${ }^{11}$ ». Pourtant, dans ses efforts pour combattre l'oisiveté, saint Augustin réhabilite le travail manuel ${ }^{12}$. Les bénédictins considèrent aussi le travail comme une pénitence, mais voient plutôt dans les tâches manuelles un moyen d'éviter le danger d'otiositas, la source du mal par excellence ${ }^{13}$. Or, la place du travail manuel dans la vie d'un moine n'allait pas de soi. Interprétant différemment les préceptes de l'évangile ou de Paul, des courants de pensée se sont alors opposés. Le moine doit-il être comme les oiseaux du ciel qui " ne sèment ni ne moissonnent " et les lis des champs qui " ne filent ni ne tissent "? Certains le soutiennent en s'appuyant sur les paroles de Mt 6,26-30. Le moine aurait ainsi tout le temps pour se consacrer au «Priez sans cesse " de Paul (1 Th 5,17), incombant aux autres chrétiens de pourvoir à son entretien. Mais, de l'autre côté, Paul recommande de travailler avec ses mains et de manger le produit de son propre travail $(2$ Th 3,12$)$. Voilà un dilemme que les règles religieuses ont tranché en essayant de trouver un équilibre entre les deux : la journée serait répartie entre les heures de prière, de lecture et de méditation, et celles consacrées au travail manuel.

Thomas d'Aquin (1225-1274) élargit cette vision positive du travail. Dans ses commentaires sur les textes pauliniens (Eph 4,17-28 et 2 Th 3,7-12), qu'il reprend dans sa Summa Theologiae Ila-Ilae, q.187, a.3, saint Thomas décrit le travail non seulement comme un remède contre l'oisiveté, mais aussi comme un moyen de gagner sa vie, un frein contre la concupiscence de la chair et une source d'aumônes 14 .

les conceptions du travail en théologie catholique », Concilium 151 (1980) p. 107-117; M.D. CHENU, "Trente ans après », LumVie 124 (1975) p. 72-77; G. LeFRanC, Histoire du travail et des travailleurs. Paris, Flammarion, 1975; M.-D. CHENu, art. « Travail », Encyclopédie de la foi. Vol. IV, Paris, Cerf, 1967, p. 347-357.

11 M. -D. Chenu, art. "Travail », Encyclopédie de la Foi, Vol. IV, p. 350.

12 Saint Augustin exprime sa pensée sur le travail manuel dans un traité appelé Le travail des moines (De opere monachorum) dirigé contre des moines qui refusaient de travailler au nom du bien supérieur de la prière et du soin des âmes. À ce sujet cf. V. DESPREZ, Règles monastiques d'Occident : $I^{e}-V^{e}$ siècle, d'Augustin à Ferróol. Bégrolles-en-Mauges, Abbaye de Bellefontaine, 1980.

13 À ce sujet cf. F. THELAMON, "Sociabilité, travail et loisir dans le monachisme antique », ASSR 86 (1994) p. 183-198 et J. DuBors, « Le travail des moines au Moyen Âge », dans Le travail au Moyen Âge, p. 61-100.

14 Selon Ph. DelHAYE, "Quelques aspects de la doctrine thomiste et néothorniste du travail ", dans Le travail au Moyen Âge, p. 169, dans son Contra Gentiles III,21, saint Thomas exprimerait déjà « quelque chose de ce que nous disons aujourd'hui sur la collaboration positive que le travail permet à l'homme d'apporter à Dieu dans l'oeuvre de la création et de la conduite du monde $»$. 
Certes, ce qu'on vient de mentionner concerne surtout les tâches des moines, mais qu'en est-il du travail dans la vie quotidienne des gens?

Il ne faut pas oublier qu'à cette époque, le travail est envisagé dans le cadre d'une vision théocentrique du monde. Comme toutes les autres créatures, l'être humain a sa place dans l'ordre de l'univers. Or, dans le plan divin, le travail est nécessaire pour maintenir l'équilibre social et communautaire. Les personnes dépendent les unes des autres et de la société. En se mettant au service d'autrui et en recevant à LEUR tour des services, les individus s'accomplissent en même temps qu'ils répondent aux besoins de la communauté ${ }^{15}$. Ainsi, dans la société médiévale, le travail est considéré comme une vocarion voulue par Dieu. Cette conception du travail, où chaque vocation reçoit une signification religieuse, est confirmée par les sermons médiévaux. Par exemple, le sermon de Berthold de Ratisbonne (1210?-1272) sur les dix chœurs des anges explique que chaque personne a une vocation et une situation sociale particulières dans l'ordre que Dieu a établi, tout comme chacun des dix chœurs des anges :

Le Tour-Puissant a donc divisé et ordonné la chrétienté en six états, dont chacun est indispensable. Il a donné un métier à chacun, comme Il l'a voulu et non comme tu le veux. Tu aimerais bien être chevalier ou châtelain, mais tu dois être cordonnier, tisserand ou paysan, comme Dieu t'a créé 16 .

Pourtant, cette vision du travail, toute optimiste qu'elle soit, crée une résignation face à l'ordre établi, puisqu'elle légitime et justifie théologiquement la distribution des tâches et la répartition sociale. L'être humain n'a qu'à se conformer à la volonté de Dieu. Le caractère douloureux qui accompagne le travail est alors interprété comme une pénitence nécessaire en vue de buts célestes. La notion de travail est ainsi placée sous le signe de l'ambiguitét ${ }^{17}$. Cette ambiguité est encore visible dans les mots employés à l'époque pour décrire le travail. En effet, jusqu'aux XII ${ }^{\mathrm{e}}$-XIII ${ }^{\mathrm{e}}$ siècles, l'Occident médiéval n'a pas un mot pour ce que nous appelons travail ${ }^{18}$. Celui-ci se trouve décrit soit par labor, laborare, laboratus,

F. SCHUSSLER FIORENZA, art. cit., p. 109

16 B. Ratisbonne, Péchés et Vertus. Paris, Éditions Desjonquères, 1991, p. 26.

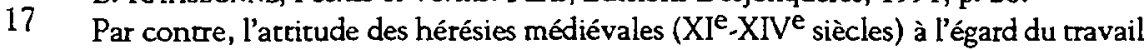
est complètement négative : les différentes formes de travail y sont condamnées. Par exemple, chez les Carhares, le travail est toléré pour ceux qui mènent une existence " attachée au mal », mais absolument interdit aux " parfaits ». C'est peut-être cette impuissance à définir une spiritualité du travail qui explique l'échec de ces mouvements (J. LE GofF, « Métier et profession d'après les manuels de confesseurs du Moyen Âge », dans Pour un autre Moyen Âge : Temps, travail et culture en Occident. Paris, Gallimard, 1977, p. 165).

18 À ce sujet cf. J. LE GofF, « Le travail dans les systèmes de valeur de l'Occident médiéval », dans Le travail au Moyen Âge, p. 7-21. 
qui évoque une notion d'effort, lequel est une conséquence du péché originel, soit par opus, opera, operatio, operari ou ars, artifex qui renvoie à une idée de création. Bien sûr, le travail comme création est essentiellement réservé à Dieu, d'où l'importance de la notion de transformation (fabricare) : le travail humain est une fabrication à partir de la matière.

\subsection{Les XVII et XVIII siècles}

La conception du travail se modifie aux XVII et et XVIIle siècles grâce aux écrits des jansénistes et des jésuites. Ceux-ci jouent un rôle de précurseurs "par rapport à la conception du travail dans la bourgeoisie et la classe moyenne ${ }^{19}$ ». La pensée janséniste place le travail sous l'angle d'une theologia crucis, c'est-à-dire de la souffrance et de la privation. La vie n'est qu'un combat de tous les instants en vue de l'élection à l'éternité. Dans cette perspective, le travail devient un moyen pénitentiel qui contribue au salut individuel, d'où l'exaltation de son aspect pénible, ennuyeux, monotone et exténuant ${ }^{20}$. Pourtant, en développant l'individualisme, le jansénisme rencontre la mentalité bourgeoise. En effet, le bourgeois est l'homme libre par excellence, tenu hors des hiérarchies seigneuriales, échappant aux liens de dépendance qui réglaient d'une manière générale les rapports entre les hommes et les biens au Moyen Âge.

De son côté, la pensée jésuite, illustrée particulièrement par les sermons de Bourdaloue (1632-1704), regarde le travail comme une "discipline ascétique ":

... car la juftice de Dieu vindicative répare le péché de l'homme par le travail, $\&$ c'eft par le travail que la juftice légale qui eft en Dieu, entretient tous les états \& toutes les conditions du monde. L'oifiveté donc qui s'oppofe directement à cette double juftice, eft un désordre 21 .

Le travail, ordonné à l'homme " comme une peine de fa désobéiffance \& de la rébellion » $(G n 3,17-18)^{22}$, n'a pas de valeur en soi. En fait, il n'a de sens que dans la mesure où il exige une vie disciplinée, laborieuse et méthodique. Cette vision du travail et de la vie fournit le cadre de formation nécessaire à la classe

20 F. SCHUSSLER FIORENZA, att. cit., p. 110.

20 La mentalité janséniste est bien illustrée par les Essais de morale de Pierre Nicole. À ce sujet cf. P. Nicole, CEuvres philosophiques et morales. Hildesheim, G. Olms Verlag, 1970. 21 Bourdaloue, « Sermon sur l'oisiveté », dans Sermons, T. 1, Nouvelle édition, Paris, G. Martin, 1750, p. 277.

22 Cette peine imposée au travail est en même temps « fatisfactoire et préfervative: fatisfactoire, pour expier le péché commis; et prefervative, pour empêcher de le commettre: fatisfactoire, parce que nous avons été prévaricateurs; \& préfervative, afin que ceffions de l'être: fatisfactoire, pour être un moyen de réparation envers la justice de Dieu; \& préfervative, pour fervir de remede à noure foi bleffé » (Ibid., 279). 
moyenne. La réussite ne se vérifie pas sans l'application indispensable au travail. Cependant, allant au-delà de la vision religieuse du travail comme simple * discipline pénitentielle ou ascétique " à valeur expiatoire, la conception bourgeoise le considère comme quelque chose de constructif qui permet le profit et le succès. Bien sûr, pour les pauvres il continue à être un châtiment du péché et un moyen de salut ${ }^{23}$, mais pour les bourgeois il est cause d'accomplissement et d'épanouissement ${ }^{24}$. On est, une fois de plus, en présence d'une ambiguité dans la conception du travail, mais cette fois-ci, elle est plutôt en rapport avec les inégalités sociales.

\section{3 À partir des années 50 et 60}

Pendant le XIX ${ }^{\mathrm{e}}$ siècle la prédication traditionnelle continue à mettre l'accent sur le « châtiment rédempteur ${ }^{25}$ " du travail. En 1942, récitant la neuvaine à saint Joseph, le croyant répétait encore :

Glorieux saint Joseph, modèle de tous ceux qui sont voués au travail, obtenezmoi la grâce de travailler en esprit de pénitence, pour expiation de mes péchés...

Pourtant, au lendemain de la Seconde Guerre mondiale, la conception religieuse du travail prend une nouvelle orientation. Les années 50 et 60 ont vu naître plusieurs tentarives de formulation d'une « théologie du travail 26 ». L'usage

23 Voir par exemple, le sermon d'Yves-Michel Marchais, curé de La Chapelle-du-Genêt, en 1773 : « Le pauvre, obligé par étar de travailler continuellement, d'agir, de s'exercer, de pourvoir à sa subsistance, est par là même bien peu occupé de plaisirs, d'amusements inutiles, et ne laisse guère de prise à l'esprit tentateur (...) Je remercie pour vous l'auteur de tous les biens qui, en vous refusant les autres, vous accorde le plus essentiel * (J. LEBRUN, « La prédication au $18^{\mathrm{e}}$ siècle $»$, dans Histoire vécue du perple chrétien. Vol. 2, Toulouse, Privat, 1979, p.61).

24 Pour la tradition puritaine le travail efficace est compris comme réponse à une vocation. Si on s'y livre de façon rigoureuse on peur s'atrendre à la réussite remporelle. Celle-ci est signe de la bienveillance divine et, dans certaines formes " abâtardies ", promesse du salut éternel. Sur ce sujet cf. M. MIEGGE, Vocation et travail. Essai sur l'éthique proritaine. (Histoire et société, 16), Genève, Labor et Fides, 1989 et P. Besnard, Protestantisme et capitalisme. La controverse post-weberienne. Paris, Colin, 1970.

25 . Voir par exemple L. LABERTHONNIËRE, Essais de philosophie religieuse. Paris, Lethielleux, 1903, p. 233-289.

26 Vour entre autres, J. THOMAS, « Une théologie du travail », Rerue de l'action populaire 164 (1963) p. 5-19; P. DE SURGY, «Élements pour une théologie biblique du travail », Masses ouvrières 165 (1960) p. 5-12; H. ROLLET, Le travail, les ouvriers et l'Église. Paris, Fayard, 1959; M.-D. CHENu, Pour une théologie du travail. Paris, Seuil, 1955; H. RONDET, « Éléments pour une théologie du travail ", NouvReuThéol 77 (1955) p. 27-48; J. LACROIX, « La notion de travail », La vie intellectuelle (1952) p. 4-31. 
même de l'expression « théologie du travail » répond déjà à un nouveau souci : dire l'honneur du travail. En effet, la théologie, "discours à l'honneur de Dieu, doit aussi dire l'honneur de l'homme créateur 27 ». En 1965, la Constitution Gaudium et Spes de Vatican II met déjà en relief la participation de l'homme, par son travail, à la création divine :

Par son travail, l'homme assure habituellement sa subsistance et celle de sa famille, s'associe à ses frères et leur rend service, peut pratiquer une vraie charité et coopérer à l'achèvement de la création divine. Bien plus, par l'hommage de son travail à Dieu, nous tenons que l'homme est associéà l'œuvre rédemptrice de Jésus-Christ, qui a donné au travail une dignité éminente en œuvrant de ses propres mains à Nazareth (GS § 67).

En 1967, l'encyclique Populorum Progressio de Paul VI reprend ces idées et les approfondit :

[Le travail] est voulu et béni de Dieu; l'homme doit coopérer avec le Créateur à l'achèvement de la création... Penché sur une matière qui lui résiste, le travailleur lui imprime sa marque, cependant qu'il acquiert ténacité, ingéniosité et esprit d'invention. Bien plus, vécu en commun, dans l'espoir, la souffrance, l'ambition et la joie partagés, le travail unit les volontés, rapproche les esprits, et soude les cœurs : en l'accomplissant, les hommes se découvrent frères (PP § 27).

L'encyclique Laborem Exercens de Jean-Paul II (1981) consacre sa dernière partie aux dimensions spirituelles du travail. C'est la première fois qu'on introduit un thème semblable dans un discours sur la doctrine sociale, bien que celle-ci soit morale et religieuse. Ces éléments spirituels du travail s'articulent autour de trois axes fondamentaux : le travail y est considéré comme participation à l'œuvre du Créateur, comme union au Christ travailleur et comme association au mystère pascal.

Le premier axe de cetre spiritualité fait appel au récit de la création de $\mathrm{G} n 1$ qui nous est présenté comme "le premier "évangile du travail" » (LE § 5,25). L'évangile est la bonne nouvelle de la vie. Crééà " l'image de Dieu ", l'être humain participe par son travail à l'activité créatrice de Dieu en exprimant et en développant la vie. Cette spiritualité du travail s'inscrit ainsi dans une perspective de vie et de joie liée à une théologie de la création.

Le deuxième axe se base sur l'exemple de Jésus travailleur. Non seulement il " a consacré la plus grande partie de sa vie sur la terre au travail manuel, à son établi de charpentier » (LE § 2,6), mais il utilise le travail humain comme parabole 
de l'agir de Dieu dans la vie des gens. Par sa vie et son enseignement, le Christ " apporte la plénitude de l'évangile du travail 28 ". Les exhortations de Paul vont dans le même sens.

Le troisième axe s'inscrit dans le mystère pascal. Le travail, par les peines qu'il comporte inévitablement ( $G n$ 3,17-19; Qo 2,11) et par sa participation au perfectionnement humain et à la transformation du monde, devient un des moyens privilégiés de contribution de l'homme à l'œuvre de salut :

En supportant la peine du travail en union avec le Christ crucifié pour nous, l'homme collabore en quelque manière avec le Fils de Dieu à la rédemption de l'humanité (LE § 5,27).

Nous voyons ainsi dans la spiritualité de Jean-Paul II que « la co-création avec Dieu Créateur et la co-rédemption avec le Christ Rédempteur ${ }^{29}$ » sont intimement liées.

\section{Conclusion}

Dans la première partie de cette étude nous avons remarqué comment les textes bibliques sont discrets. Ils ne donnent pas beaucoup d'informations sur la manière de travailler dans chacun des métiers et ne présentent pas de grands discours sur la valeur et la signification du travail. Cependant, le travail y est à l'honneur. Notre analyse du vocabulaire et de quelques textes bibliques de la Genèse, des Sages, des Prophètes et du Nouveau Testament nous a bien montré que la conception optimiste et épanouissante du travail traverse toute la Bible. Si parfois le travail y est décrit comme une tâche pénible, ce n'est pas à cause d'une malédiction quelconque, mais à cause de la finitude humaine (Qo) et de la désobéissance de l'être humain aux commandements de Dieu $(G n 3,17-19)$. Les rapports humains sont alors envenimés par l'arbitraire, l'injustice, l'exploitation, le mensonge et l'ambition démesurée.

Dans la deuxième partie de cette étude, nous avons tenté de montrer comment la tradition chrétienne occidentale, jusqu'à une époque bien récente, avait plutôt mis l'accent sur le caractère pessimiste du travail. Certes, on a décrit le travail comme étant une vocation divine et contribuant au maintien d'une certaine cohésion sociale. Pourtant, une conception péjorative du travail, l'assimilant surtout à une malédiction et un châtiment du péché, a servi à justifier une attitude de laisser-faire à l'égard de l'ordre établi. En effet, face aux peines et aux souffrances du travail, on a toujours proclamé qu'il fallait non seulement s'incliner, mais remercier Dieu, car elles étaient instruments de rédemption. 
En réaction contre ce pessimisme, un changement s'opère, à partir des années 50 et 60 , dans la perception théologique du travail. Désormais on reconnaîtra dans celui-ci non seulement une collaboration à l'œuvre salvifique du Christ, mais surtout une participation à l'œuvre créatrice de Dieu. Par son travail, l'être humain n'est-il pas appelé à exercer sur soi-même, sur son entourage et sur son environnement, cette harmonie qui témoigne de la bénédiction du Créateur? Or cette conception optimiste du travail n'est pas sans nous interroger. Dans une société où les emplois sont de plus en plus précaires, nous disons avec les Évêques des États-Unis : « le travail étant si important, les hommes et les femmes ont droit à l'emploi ${ }^{30}$ ». sur l'enseignement social catholique et l'économie américaine. Paris, Cerf, 1988, p. 96. 